\title{
Biomarker microfibril-associated glycoprotein 4 for non-invasive diagnosis and therapeutic evaluation of hepatic fibrosis in patients with hepatitis $\mathrm{C}$
}

\author{
Young Woo Eom ${ }^{1}$ and Soon Koo Baik ${ }^{2}$ \\ ${ }^{1}$ Cell Therapy and Tissue Engineering Center and ${ }^{2}$ Department of Internal Medicine, Yonsei University Wonju College of Medicine, \\ Wonju, Korea
}

Keywords: Biopsy; Serologic tests; Fibrosis; Hepatitis C

See Article on Page 42

Cirrhosis represents the end stage of chronic liver disease, which may cause severe hepatic dysfunction, portal hypertension, and eventually progress to hepatocellular carcinoma. ${ }^{1-3}$ Cirrhosis is characterized by fibrosis and the conversion of normal liver architecture into abnormal nodules surrounded by annular fibrosis. ${ }^{4}$ Therefore, accurate assessment of the degree of fibrosis and/or apparent cirrhosis is necessary for accurate diagnosis and decision-making for optimal treatment, which consequently determines patients' prognosis. Liver biopsy remains the gold standard to determine the degree of hepatic fibrosis. However, accurate diagnosis and periodic assessment to confirm cure are difficult owing to limitations including sampling errors, inter-observer variability, and invasiveness of this diagnostic technique., ${ }^{5,6}$ Therefore, non-invasive modalities including diagnostic ultrasonography and transient elastography (TE) and serological tests are useful alternatives to liver biopsy forassessing hepatic fibrosis and cirrhosis. ${ }^{7-10}$
Serological markers can be classified as direct or indirect markers. ${ }^{5}$ Direct markers are derived from the extracellular matrix or secreted by activated hepatic stellate cells. ${ }^{11-13}$ Hyaluronic acid (HA), procollagen type I carboxy-terminal peptide, procollagen type III amino-terminal peptide, metalloproteinases, tissue inhibitors of matrix metalloproteinases, laminin, transforming growth factor-1, connective tissue growth factor, cartilage glycoprotein-39, microfibril-associated glycoprotein 4 (MFAP4), and cytokeratin-18 fragments are currently used as direct serum markers of hepatic fibrosis. Indirect markers include serum aspartate aminotransferase and gamma-glutamyl transferase produced by hepatocytes and bile duct cells in the liver parenchyma after chronic liver injury, serum bilirubin indicating impaired liver's capacity to transport organic anions, and platelets and serum immunoglobulin-gamma indicating the presence of portal hypertension. ${ }^{5}$ Serological testing (using direct and indirect markers) is non-invasive. Thus, it can be used periodically, alone or in combination with other modalities to easily and accurately assess the degree of improvement in fibrosis in patients receiving treatment for cirrhosis.

\section{Abbreviations:}

$H A$, hyaluronic acid; MFAP4, microfibril-associated glycoprotein 4; TE, transient elastography

\section{Corresponding author : Soon Koo Baik}

Department of Internal Medicine, Yonsei University Wonju College of Medicine, 20 Ilsan-ro, Wonju 26426, Korea

Tel: +82-33-741-0503, Fax: +82-33-741-1228

E-mail:baiksk@yonsei.ac.kr

https://orcid.org/0000-0001-6245-2537 
Following proteomic analysis of microdissected cirrhotic septa and liver parenchyma cells, MFAP4 was identified as a new biomarker for hepatic fibrosis in patients with cirrhosis. ${ }^{14}$ MFAP4 is strongly expressed in cirrhotic septae, but not in a normal liver. Additionally, previous large-scale studies have reported that serum levels of MFAP4 showed high diagnostic accuracy as predictors of normal vs. cirrhotic liver (area under receiver operating characteristic curve $=0.97, P<0.0001$ ), as well as stage 0 vs. stage 4 fibrosis (area under receiver operating characteristic curve $=0.84$, $P<0.0001$ ), and stages $0-3$ vs. stage 4 fibrosis (area under receiver operating characteristic curve $=0.76, P<0.0001)^{14}$

In the current issue, Mölleken et al. assessed the usefulness of serum levels of MFAP4 as a biomarker of hepatic fibrosis after treatment of hepatitis $C$ virus infection with direct acting antivirals..$^{15}$ The authors measured serum levels of MFAP4 using an immunoassay in 50 patients with hepatitis $C$ infection at baseline, at the end of therapy, and at the 12-week follow-up. ${ }^{15}$ The study analyzed the association between serum MFAP4 levels and laboratory parameters including the aspartate aminotransferase-toplatelet ratio index and serum levels of alanine aminotransferase, aspartate aminotransferase, platelets, and albumin. ${ }^{15}$ Serum levels of MFAP4 were associated with the severity of hepatic fibrosis at baseline and were significantly associated with laboratory parameters, particularly with the aspartate aminotransferase-to-platelet ratio index (Spearman correlation, $\mathrm{R}^{2}=0.80$ ). Monitoring of laboratory parameters showed that levels measured at the end of treatment were significantly lower than those measured at baseline. Serum levels of MFAP4 decreased from baseline and the end of treatment until follow-up and this difference was statistically significant (Wilcoxon $P<0.001$ for both time-points). They observed that viral eradication caused a reduction in the serum levels of MFAP4, which reflected a decrease in hepatic fibrogenesis or fibrosis. ${ }^{15}$ Thus, the study reported by Mölleken et al. suggested that serum levels of MFAP4 may be a useful tool to assess the risk of patients with hepatitis $C$ with advanced fibrosis after virus eradication. ${ }^{15}$

It is reasonable to conclude that the MFAP4 protein would be a useful biomarker to assess the degree of hepatic fibrosis. However, it is necessary to use combinations of MFAP4 and other serum markers or imaging techniques for better diagnostic accuracy for hepatic fiborsis. Lee et al. assessed and compared serum levels of HA and cartilage glycoprotein-39, as well as findings of TE as predictors of advanced hepatic fibrosis in a single-center pediatric cohort. ${ }^{7}$ In predicting hepatic fibrosis, HA and TE were found to have significant concordance with fibrosis stage, but TE was su- perior to HA. However, the addition of HA as a serum biomarker to TE did not improve the performance of predictability of TE for hepatic fibrosis. Moreover, serum levels of cartilage glycoprotein-39 showed no predictive performance for hepatic fibrosis. Thus, the authors suggested that TE and HA may be useful noninvasive tools to assess the degree of hepatic fibrosis in children. ${ }^{\text {? }}$ Lannerstedt et al. evaluated the predictive value of TE and other easily available biochemical scores including the aspartate aminotransferase-to-platelet ratio index and the fibrosis- 4 scores, and the aspartate aminotransferase/alanine aminotransferase ratio, as well as a combination of these parameters to predict advanced hepatic fibrosis in 418 patients with chronic liver disease attributable to different etiologies. ${ }^{8}$ The authors suggested that the combination of TE and the fibrosis- 4 score was useful to predict advanced fibrosis, although the predictive performance effect of this combination for hepatic fibrosis was insignificant when testing was performed in only asymptomatic patients. ${ }^{8}$

In conclusion, a single non-invasive serological test or imaging technique is incapable of assessing the progression of fibrosis and can therefore not replace liver biopsy as a diagnostic modality. To overcome this limitation, a greater number of large-scale studies are warranted to determine the optimal combination of non-invasive serological tests and imaging techniques to reliably and accurately diagnose and evaluate the progression of fibrosis.

\section{Authors' contribution}

Manuscript writing: Young Woo Eom and Soon Koo Baik

Final approval of manuscript: Young Woo Eom and Soon Koo Baik

\section{Conflicts of Interest}

The authors have no conflicts to disclose.

\section{REFERENCES}

1. de Franchis R. Evolving consensus in portal hypertension. Report of the Baveno IV consensus workshop on methodology of diagnosis and therapy in portal hypertension. J Hepatol 2005;43:167-176.

2. Bruix J, Sherman M; American Association for the Study of Liver Diseases. Management of hepatocellular carcinoma: an update. Hepatology 2011;53:1020-1022.

3. European Association For The Study Of The Liver; European Organisation For Research And Treatment Of Cancer. EASL-EORTC clinical practice guidelines: management of hepatocellular carcinoma. J 
Young Woo Eom, et al. MFAP4 for non-invasive biomarker of hepatic fibrosis

Hepatol 2012;56:908-943.

4. Anthony PP, Ishak KG, Nayak NC, Poulsen HE, Scheuer PJ, Sobin LH. The morphology of cirrhosis. Recommendations on definition, nomenclature, and classification by a working group sponsored by the World Health Organization. J Clin Pathol 1978;31:395-414.

5. Soresi M, Giannitrapani L, Cervello M, Licata A, Montalto G. Non invasive tools for the diagnosis of liver cirrhosis. World J Gastroenterol 2014;20:18131-18150.

6. Mehta SH, Lau B, Afdhal NH, Thomas DL. Exceeding the limits of liver histology markers. J Hepatol 2009;50:36-41.

7. Lee CK, Perez-Atayde AR, Mitchell PD, Raza R, Afdhal NH, Jonas $M M$. Serum biomarkers and transient elastography as predictors of advanced liver fibrosis in a United States cohort: the Boston children's hospital experience. J Pediatr 2013;163:1058-1064.e2.

8. Lannerstedt H, Konopski Z, Sandvik L, Haaland T, Løberg EM, Haukeland JW. Combining transient elastography with FIB4 enhances sensitivity in detecting advanced fibrosis of the liver. Scand J Gastroenterol 2013;48:93-100.

9. Islam S, Antonsson L, Westin J, Lagging M. Cirrhosis in hepatitis C virus-infected patients can be excluded using an index of standard biochemical serum markers. Scand J Gastroenterol 2005;40:867-872.

10. Lok AS, Ghany MG, Goodman ZD, Wright EC, Everson GT, Sterling $\mathrm{RK}$, et al. Predicting cirrhosis in patients with hepatitis $\mathrm{C}$ based on standard laboratory tests: results of the HALT-C cohort. Hepatology 2005;42:282-292.

11. Gressner AM, Haarmann R. Hyaluronic acid synthesis and secretion by rat liver fat storing cells (perisinusoidal lipocytes) in culture. Biochem Biophys Res Commun 1988;151:222-229.

12. Schuppan D, Stölzel U, Oesterling C, Somasundaram R. Serum assays for liver fibrosis. J Hepatol 1995;22(2 Suppl):82-88.

13. Gressner OA, Weiskirchen $R$, Gressner AM. Biomarkers of liver fibrosis: clinical translation of molecular pathogenesis or based on liverdependent malfunction tests. Clin Chim Acta 2007;381:107-113.

14. Mölleken C, Sitek B, Henkel C, Poschmann G, Sipos B, Wiese S, et al. Detection of novel biomarkers of liver cirrhosis by proteomic analysis. Hepatology 2009;49:1257-1266.

15. Mölleken C, Ahrens M, Schlosser A, Dietz J, Eisenacher M, Meyer $H E$, et al. Direct-acting antivirals-based therapy decreases hepatic fibrosis serum biomarker microfibrillar-associated protein 4 in hepatitis C patients. Clin Mol Hepatol 2019;25:42-51. 